\title{
An Overview of Healthcare Information Technologies Used to Combat the COVID-19 Pandemic in China
}

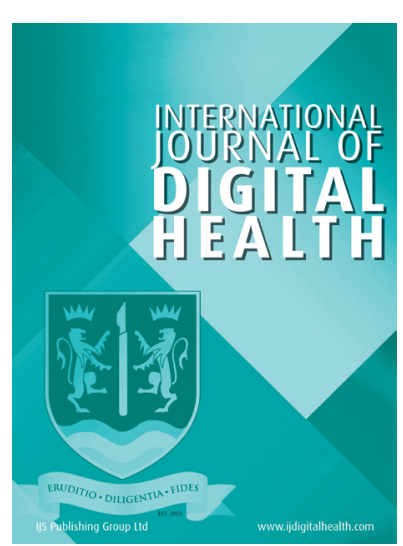

JILAN LIU

ZHIMOU WANG

SIYUN HUANG

ANJIE REN (D)

*Author affiliations can be found in the back matter of this article

\begin{abstract}
This article describes the rapid development and deployment of healthcare information technologies (HIT) in response to COVID-19 from late January through March 2020 in China. It outlines the broad scope and in-depth penetration of various products and services, as well as the impacts that these products/services had in reaching the mass population for effective information dissemination, contact tracing, early diagnosis, virtual care, teleconsultation, social distancing and community outreach and support. The article also describes the rapid development of new infrastructures and the exponential growth of usage in the various technologies. Combined with other measures, HIT have played a crucial role in China through galvanizing public response in solidarity and achieving a massive scale of contact-tracing and interventions in a personalized manner.
\end{abstract}

\section{CORRESPONDING AUTHOR:}

\section{Jilan Liu}

HIMSS Greater China, Seattle, USA

jilanliu@himss.cn

\section{KEYWORDS:}

Big data; China; contact tracing; COVID-19 pandemic; digital technology; healthcare information technology; telehealth

TO CITE THIS ARTICLE:

Liu J, Wang Z, Huang S, Ren A. An Overview of Healthcare Information Technologies Used to Combat the COVID-19 Pandemic in China. International Journal of Digital Health. 2021; 1(1): 14, 1-9. DOI: https://doi.org/10.29337/ ijdh.37 


\section{INTRODUCTION}

The outbreak of pneumonia caused by COVID-19, first reported in Wuhan, China in December 2019, was quickly discovered in the rest of the country and globally. This pandemic became one of the biggest problems devastating the entire world in 2020-2021.

From January 19, 2020, when the coronavirus was confirmed to be transmissible among people, China went on an all-out war against the spread of the virus and it took less than a month to start seeing the flattening of the curve. By March 2020, COVID-19 was effectively under control in China with no more than occasional three-digit numbers of weekly new cases until now [1]. Many measures contributed to the success of China's speedy response. Among them, the effective use of information technologies is the one shared in this article.

There were about 1.6 billion mobile subscriptions in China by December 2019 [2]. The predominance of mobile phone use in China prior to and during COVID-19 provided a solid foundation from which digital solutions could be built for the mass population. Development and implementation of healthcare information technologies (HIT) in recent years had significantly elevated its maturity level in China, which in turn had improved the country's response capacity to the COVID-19 outbreak. For example, China had the 2nd highest number of HIMSS (Health Information and Management Society) EMRAM (Electronic Medical Record Adoption Model) Stage 7 Hospitals in the world, next only to the United States [3]. HIT had also played a pivotal role in China's rigorous response measures, such as proactive surveillance for rapid detection and diagnosis of infection, immediate isolation, contact tracing, quarantine of close contacts, and exceptionally high awareness and acceptance of these measures among the general public. According to the Report of the WHO-China Joint Mission on Coronavirus Virus (COVID-19), China's bold approach to contain the rapid spread of this new coronavirus had changed the course of a rapidly escalating and deadly pandemic [4].

The emergence of innovative developments and uses of information technologies in China's fight against the COVID-19 epidemic was unprecedented. China fought hard and was effective in bringing COVID-19 under control with the aid of information technologies. The following examples illustrate how China had harnessed HIT to tackle the pandemic from January to March 2020.

\section{1) ONLINE PANDEMIC INFORMATION DISSEMINATION PLATFORMS}

Shortly after the outbreak, Chinese media in both the public and private sectors created platforms to increase awareness and release updates about the epidemic. At least five major official and commercial news websites and smart phone apps had been extensively accessed by people seeking up-to-date pandemic information, including the People's Daily [5], Dingxiangyuan (DXY, an Internet-based health news agency) [6], Alipay [7], Tencent [8], and Netease [9]. The figures included daily updates on new and cumulative numbers of confirmed, suspected and critically ill cases, death tolls nationwide and by province and city, as well as global statistics, which were sourced from China's National Health Commission and the local health and disease control authorities. The online media also provided a variety of useful information and tools, such as color-coded epidemic maps, trend curves, COVID-19 news stories, disease prevention and control knowledge, 'Fact Check' guides for refuting rumors and correcting misinformation, and free COVID-19 consultations. Some online platforms had even offered custom features such as 'My Epi Updates' for users to personalize their own daily updates about the pandemic status in the cities of their interest, and 'Rides Look-up' for travelers to determine if they had shared transit rides with any confirmed case. Other popular platforms included 'Cases Nearby' and 'Cases in My Neighborhood' [10], which identified whether the reported locations of the confirmed cases were close to the user's current location, and displayed the areas that the confirmed cases had gone to based on cellphone base station data, without disclosing any personal information of the individuals. Users could readily find common pandemic-related information on one of these all-in-one information platforms. In fact, these platforms had become so popular that the Dingxiangyuan platform had received over 2.5 billion visits by February 27, 2020 [6]. The role that such platforms played in informing the public during the COVID-19 pandemic was tremendous and contributed to enhancing the awareness, understanding and solidary support among the Chinese population of 1.4 billion.

\section{2) ARTIFICIAL INTELLIGENCE (AI)- ASSISTED INFECTION RISK IDENTIFICATION, TEMPERATURE MONITORING, ONLINE SCREENING, AND CONSULTATION PLATFORMS}

Internet hospitals and cloud-based medical consultation platforms owned by businesses and hospitals launched free online consultation services soon after the outbreak to help the general public screen for possible infections. Demand for cloud consulting and tele-medicine providers surged during this period. For example, Hangzhou Joinhealth Technology Co., Ltd. had quickly developed an AI tracking platform for people at risk for COVID-19. The platform provided self-registration and crowd management modules for the at-risk populations, such as people who flew out of Wuhan, those in close contact with an infected person, those on home isolation, and those having a fever [11]. Integrated with a smart followup platform which had been operated by Joinhealth for many years, the AI tracking platform was interfaced with over 400 hospitals for sharing information and following 
up the daily health status of high-risk groups. The 'Manniu Health' team under the Wonders Information Technology Co. Ltd had also developed a free online screening toolkit called the 'COVID-19 Quick Test'. The team had compiled clinical guidelines and epidemic investigation strategies, and employed big data and AI technologies to build the toolkit, which helped residents quickly learn their health status, infection risks and the recommendations offered by the toolkit according to the screening results [12].

Self-screening features on smartphone apps were helpful tools for early detection of COVID-19 infections and initiating care. On February 26, 2020, the health authorities in Wuhan started recommending residents to use online screening apps. Changsha, capital city of Hunan province, launched a COVID-19 early selfscreening feature on their "My Changsha" app, the city's official integrated service platform. Shanghai and other major Chinese cities had also initiated similar efforts [13]. By responding to a set of simple questions, users could receive an instant notification about their infection risks with AI-generated personalized recommendations, such as 'Keep up with the strict precautions to minimize risks', 'Immediate self-isolation is required', 'Please report to the local community authority', and 'Please go to a hospital for immediate medical attention', among other responses. Furthermore, some apps could even provide specific instructions to the user, such as how to take precautions, how to self-isolate for 14 days, or where to find a fever clinic or a designated hospital for COVID-19. In addition, the Tsinghua University Institute of Precision Medicine, Institute of Artificial Intelligence, and Beijing Tsinghua Changgung Hospital, the affiliated hospital of Tsinghua University had jointly developed and launched the COVID-19 Self-Screening System on February 1, 2020, for free public access [14].

AI was also used to perform contract tracing and tracking based on big data generated by cell phones, transportation records and online payments to help disrupt coronavirus transmission. The data were initially used to trace people who went out of Wuhan. Later on they were used to locate individuals who shared the same train, flight, bus, or taxi with a confirmed patient. These individuals could be tracked down and contacted anywhere in China. For example, a taxi driver in Zhejiang province was diagnosed with COVID-19. Most of his passengers within 14 days before his diagnosis were identified with their digital payment records. The other nine passengers who paid by cash reported themselves to local authorities after they saw the trip details published on public media [15]. Trains, the main mode of transport in China, carried approximately three billion passengers during the 2019 Spring Festival period. All train passengers were required by the Chinese railroad authority to register their personal cell phone numbers when they purchased tickets [16]. This facilitated the contact tracing and contacting of the passengers who were suspected of infection or had shared a ride with an infected individual. Used together with the national digital railway ticketing system, transit-based big data became a powerful tool for tracking down and tackling possible coronavirus transmissions.

In regard to symptom monitoring, high-performance infrared thermal imaging was used to monitor fever, which was the most common symptom of COVID-19 infection. This form of monitoring allowed rapid measurements of body temperatures of persons in crowds, even in constant motion. High-performance infrared thermal cameras were installed at the entrances of train stations and airports and other major public facilities. The devices were able to capture thermal images of mass groups of people in real time and rapidly detect persons with abnormal temperatures. Unlike conventional manned temperature taking, the technology could work continuously $24 / 7$ and as a result tremendously enhanced detection efficiency and significantly reduced the risk of transmission via human contact. Technology companies were also proposing the use of AI to establish the identity of passengers in the measurement area, which would allow those suspected of infection to be immediately isolated from the crowd [17]. In order to allow front-line disease control staff to measure body temperature without physical contact, Da-Jiang Innovations (commonly known as DJI), a world leading drone developer, launched an emergency temperature measurement solution for their drones. By modifying their drones with a cotton swab, DJI could improve the accuracy of their drones up to $\mathrm{a} \pm 0.5^{\circ} \mathrm{C}$ margin of error when monitoring for fever in individuals [18]. Drones that were equipped with an infrared thermometer were used to measure body temperatures of residents with no need for them to leave their homes in some neighborhoods. The person whose temperature was to be measured would be instructed by a loudspeaker mounted on the drone to step closer to an open window for temperature taking, without any physical contact in the process [19].

With the vast numbers of people under quarantine or self-isolation during COVID-19, many of them needed professional psychological counseling. A number of online tele-medicine companies, such as Chunyu Yisheng, worked with hospitals to launch free Internet-based consultation services within days after the pandemic began. Other organizations and companies included WeDoctor, which also started to provide psychological counseling online; and Xikang Cloud Hospital, which offered free online COVID-19 consultation. In addition, Zhuojian China provided free imaging consulting services for Internet hospitals [20]. Peking University Health Information Technology Co., Ltd. (PKU-HIT) helped hospitals deploy new online fever consultations that can be launched in less than 6 hours free of charge [21]. China Mobile devised free remote consultation 
platforms for medical teams in Guangxi, Shanghai and Jiangsu and helped provide 5th Generation (5G)-based remote diagnosis and treatment, screening and thermal imaging in Chongqing, Sichuan and Zhejiang. Other online counseling apps, such as the popular 'Healthy Living Club', was also specially developed for individuals who were isolated because of the pandemic.

\section{3) AI-ASSISTED RADIOLOGICAL IMAGE INTERPRETATION AND INTERVENTION RECOMMENDATIONS}

During the pandemic, AI-assisted image reading programs were launched to help diagnose COVID-19 patients more quickly. A cloud-based coronavirus pneumonia diagnosis service providing AI-assisted computed tomography (CT) image interpretation was launched by Huawei Cloud, a healthcare business branch of Huawei [22]. The Public Health Center of Shanghai had validated a similar technology developed by YITU Tech, a Shanghai company specializing in health AI, which could read lung images with an accuracy equivalent to an experienced radiologist [23]. Both technologies were capable of processing large numbers of CT images in seconds and speed up diagnosis significantly. Winning Health, an electronic medical record (EMR) company in Shanghai, released a cloud-based AI-assisted diagnosis assistant for COVID-19, providing free online screening and diagnosis services for physicians and radiologists to identify suspected COVID-19 cases more efficiently. The product was adopted by major counter-epidemic operations, such as the Henan province's version of 'Xiaotangshan Hospital' [24]. Similar products and services were also launched by technology giants such as Tencent.

\section{4) COMMUNITY OUTREACH AND SUPPORT}

Huangpu district of Guangzhou city, Guangdong province launched a Huangpu District Epidemic Prevention and Control Platform [23] to keep track of community risks and provided support to individuals who needed to be isolated during COVID-19. With the app, residents were able to submit daily health status reports online, which were then reviewed by their 'grid health manager', a community worker designated for a section of the district, who would respond with a message, a phone call, or even a home visit if necessary. The latest addition to the app was an 'I Need' feature for residents who were isolated in their homes for suspected infections [25]. This feature allowed the user to type in their needed daily necessities, like rice, meat, vegetables, and medications. The community officials would then help arrange to buy the items and deliver them to the isolated residents after receiving the message on the app. Yan'an city, Shaanxi province had a city-wide integrated urban administration system known as the 'City Cloud Brain', which incorporated over 1,300 video feeds from public stationary cameras, AI-powered surveillance cameras, drone-borne cameras, and portable digital recorders to monitor crowd gathering in public areas and help dispatch police officers for intervention [14].

Based on the national integrated government service platform, Alipay developed an epidemic prevention and control health quick response (QR) code system in spring 2020, which was interfaced with Dingtalk, Alibaba's telecommuting platform for companies. The "Health QR Code" was a COVID-19 risk status code, which was generated based on the data from a standard questionnaire about a person's recent possible exposures and risks. The QR code became a digital 'passport' for local residents and migrant workers when they entered and exited a city [26]. The three-color traffic light system was used for coding a user's risk level, i.e. green, yellow and red for low, medium and high risk respectively, which dictated the user's travel restriction level accordingly. Users with a green code were approved to board all public transit, while users with a red or yellow code had to self-isolate at home for 14 or 7 days. Tencent WeChat also launched a similar feature on their miniapp to help individuals and communities through a three-colored online health certificate. Furthermore, the Wonders Group developed a 'personal code' for Shanghai residents on a local app which updated the user's health status regularly [27]. In addition to travel, these QR code systems were also used for monitoring in many public venues such as stores where users had to scan a location QR code to generate an entry permit.

\section{5) BIG DATA ANALYTICS FOR PREVENTION AND CONTROL OF THE PANDEMIC}

a) Predictive modeling and turning point projection

The power of big data was clearly demonstrated in China's fight against COVID-19. Many technologies were built on the basis of big data, which became an asset for tackling COVID-19. By aggregating and analyzing the pandemic data with advanced analytic techniques for big data and cloud computing, public health authorities were able to optimize their decisionmaking. Local authorities used big data tools like Baidu Migration Map to monitor how many people came into cities or left for the rural areas. Based on the migration patterns and population distribution, local authorities were able to predict the number of potentially infected individuals and hence decide supply allocation and implementation of control measures [28]. Additionally, prediction models were established to estimate the distribution of asymptomatic patients and predict the likely turning point of the pandemic after factoring in other data, such as trends in confirmed and emerging cases. Nankai University was one of the institutions that offered data support for pandemic prevention and control with regular predictive analyses [29]. 


\section{b) Supercomputing for vaccine and drug development}

Several supercomputer centers in China were working to assist the China Center for Disease Control (CDC) to develop new vaccines. Researchers in China released the genetic sequence of the 2019-nCOV during the early stage of the pandemic. Supercomputers were used for target exploration, drug selection, primer and experiment optimization, pharmacological and toxicological studies. Designated laboratories which received the viral strains began vaccine research and development and drug selection [14]. Alibaba opened up its computing power to the global research community free of charge [30].

\section{6) TECHNOLOGIES THAT SUPPORTED SOCIAL DISTANCING}

\section{a) Internet Plus (Internet+) medicine}

During COVID-19, many online healthcare and telemedicine platforms saw unprecedented growth. Most routine hospital visits for chronic disease followups, drug refills, and elective hospital admissions were put on lower priority to free up resources for the care of COVID-19 patients and minimize risks of cross-contamination in over-crowded hospitals [31]. Independent Internetbased healthcare providers and Internet hospitals that were affiliated to a physical hospital introduced a variety of measures to increase online registrations and consultations. Winning Health [32], a HIT developer based in Shanghai with an online consultation product line, experienced an increased demand since the beginning of the pandemic. Ewell, another HIT company in Hangzhou, also deployed an online consultation system for a number of hospital clients, including MDT (multidisciplinary team) platforms for remote multi-disciplinary consultations for critically ill cases [33]. An online health consultation application from the PingAn Group, one of China's largest health systems, reported a 5-10 fold increase in the use of online registrations and consultations [34]. On February 23, 2020 Shanghai Medical Insurance Authority announced the 'Twelve Measures for Medical Insurance' after the Zhejiang Provincial Medical Insurance Authority took the lead to include Internet+ healthcare services for medical insurance reimbursements [35].

\section{b) Tele-medicine services}

Major hospitals in China had long established telemedicine mechanisms with medical groups or Internet hospitals pre-COVID-19. During the pandemic, telemedicine had found an even broader use in China. Ngari Health collaborated with the Wuhan Huoshenshan Hospital to complete the commissioning of a remote consultation platform [36]. A COVID-19 medical team in Guangdong province held online multi-disciplinary consultation tele-conferencing sessions with experts in other cities [24]. Experts from the West China Hospital of Sichuan University could view and discuss lung images of
COVID-19 cases with physicians in Wuhan via a 5G-based tele-medicine platform [37]. The First Affiliated Hospital of Zhongshan University offered multi-disciplinary consultations with hospitals in Wuhan where more than 30 experts from over 10 specialties engaged in discussions of critically ill COVID-19 cases [38].

\section{c) Telecommuting and online education}

The COVID-19 outbreak started just before the beginning of China's Spring Festival (Lunar New Year) holiday season, which was the country's most celebrated and longest holiday and where there was a large population migration over this brief period. This holiday season was officially extended twice, initially by one week and then by another two weeks, as most businesses and all schools across the country feared the virus spreading. All citizens, except those engaged in the COVID-19 response, were required to stay home and away from crowds. With the back-to-work and back-to-school dates postponed, telecommuting and online education providers found unprecedented opportunities for business growth. Telecommuting became a new norm for businesses during the pandemic. To minimize delay of classes for students, many Chinese online education providers also provided livestreaming classes and opened up their recorded lessons free of charge. Online ordering, gaming and many other Internet-based industries also experienced unprecedented growth. DingTalk, the telecommuting platform of Alibaba [39], WeChat of Tencent [40], and Troila Technology [41] even promised free-of-charge services until the end of the pandemic.

\section{d) Drones for crowd activity monitoring}

Drones were used in China on many occasions for crowd control and monitoring during the pandemic. Police officers remotely controlled drones mounted with a camera and a loudspeaker to monitor people outdoors [42]. Individuals gathering in crowds or not wearing masks in public places between January and March 2020 received airborne instructions from these drones to disperse and go home and/or to put on a mask. The use of such drones considerably improved the monitoring and intervention efficiency with notable results.

\section{e) Contact-free take-away food delivery services}

Some of China's leading take-away food apps, such as Meituan [43] and Ele.me [44], tried out new ways of food delivery. When placing a food order, the customer could call the courier and agree on a location to drop the food, for example, outside the gate of a residential compound. The courier would give the customer a call or a message on the app after he had made the drop, and the customer would then be able to pick it up from the agreed location. This process prevented any direct physical contact between the courier and the customer. To take it to the next level, automated smart delivery robots were also 
deployed to deliver food in certain circumstances, such as a designated hospital caring for COVID-19 patients.

\section{7) 5G-BASED ROBOTICS AND INFRASTRUCTURE}

Robots were dispatched to work in hospitals during COVID-19 to perform functions such as screening and orienting patients, delivering supplies, medications and food, disinfecting spaces, and even providing company. A handful of Chinese hospitals, including the Wuhan Union Hospital, received robots tailored to the COVID-19 pandemic [45]. The use of robots in contagious environments helped reduce the chance of crossinfection and save manpower.

Wuhan, the epicenter of China's COVID-19 epidemic, was on a strict lockdown between January 23 and April 8, 2020. As the number of patients continued to rise, there were not enough beds to admit and isolate suspected or infected patients in the early stage of the epidemic. State authorities mobilized massive resources to help the city fight the virus shortly after it broke out. Two new makeshift hospitals designated for COVID-19 patients, the 1,000-bed Huoshenshan Hospital and the 1,200 -bed Leishenshan Hospital (named after God of Fire and God of Thunder in ancient Chinese mythology, who were believed to combat diseases), were built and operationalized in ten and twelve days [46]. To help increase public awareness of the additional resources being built and maintain hope, live broadcasts and streaming of the two hospitals' construction sites were released and well-received [47]. Technology companies also moved quickly. For example, Huawei completed planning, inspection, laying of optic fiber, base station installation, and commissioning of the $5 \mathrm{G}$ communications infrastructure for the Huoshenshan Hospital in three days, for teleconferencing, remote surgery and data transmission [48]. Other hospitals in Wuhan also launched EMR and network systems at an extraordinary speed. Donghua Software (DHC), a major Chinese HIT vendor, deployed the EMR system of the Huoshenshan Hospital in ten days [32].

\section{8) INTERNET-OF-THINGS (IOT) TECHNOLOGIES}

The ‘Biosafety Credibility Management Platform', a series of products from Honor Trends, a Beijing tech company specializing in smart management of frozen bioproducts, integrated IoT, AI and blockchain technologies for unique identification of clinical specimens, blood, reagents and supplies used in response to COVID-19 [49]. Another IoT technology developed was the medical waste intelligent cloud management system from Ewell, one of China's leading HIT companies in Hangzhou, which helped hospitals automatically weigh and track their medical wastes [50]. The intelligent temperature detection solution by Tencent Cloud improved efficiency and safety of temperature measurement with online resident registration, automatic temperature collection, and personal information management [51]. The temperature detection solution provided a one-stop feature to support the community's offline temperature taking processes.

\section{CONCLUSION}

This article has provided an insight into a non-exhaustive list of applications and scenarios in which China had used HIT to empower its fight against the COVID-19 pandemic between January and March, 2020. In fact, China was not alone, and we have seen the effective use of numerous HIT applications worldwide in the global fight against this pandemic. What was unique in the scenarios described in this article was the breadth, depth and speed in which the applications and services were developed and adopted to large-scale uses, sometimes in just a matter of days or weeks. This could not be possible if HIT had not been widely adopted in Chinese society and in particular the healthcare sector prior to the pandemic, in addition to having a centralized epidemic response system [52]. In essence, HIT had played a crucial role in China's response to combat the COVID-19 pandemic, with the country achieving a massive scale of contact-tracing and healthcare interventions in a personalized manner.

\section{COMPETING INTERESTS}

The author has no competing interests to declare.

\section{AUTHOR AFFILIATIONS}

\section{Jilan Liu}

HIMSS Greater China, Seattle, USA

\section{Zhimou Wang}

HIMSS Greater China, Guangzhou City, Guangdong Province, CN

Siyun Huang

HIMSS Greater China, Chengdu City, Sichuan Province, CN

Anjie Ren (D) orcid.org/0000-0002-1700-2585

HIMSS Greater China, Chengdu City, Sichuan Province, CN

\section{REFERENCES}

1. World Health Organization. China: WHO Coronavirus Disease (COVID-19) Dashboard. https://covid19.who.int/ explorer, 2021 (accessed 10 March 2021). DOI: https://doi. org/10.46945/bpj.10.1.03.01

2. Statista. Number of mobile cell phone subscriptions in China from December 2019 to December 2020. https:// www.statista.com/statistics/278204/china-mobile-usersby-month, 2021 (accessed 10 March 2021).

3. HIMSS Analytics. Stage 6 and 7 Achievements. https:// 
www.himssanalytics.org/stage-6-7-achievement, 2020 (accessed 7 February 2020).

4. World Health Organization. Report of the Chinese-WHO Joint Expert Group (Chinese version). 16-24/02/2020.

5. People's Daily. Realtime National COVID-19 Statistics. http://health.people.com.cn/GB/26466/431463/431576/ index.html, 2020 (accessed 8 February 2020).

6. Dingxiangyuan. COVID-19 Realtime Stats. https://ncov.dxy.cn/ncovh5/view/pneumonia_ peopleapp?from=timeline\&isappinstalled=0, 2020 (accessed 10 February 2020).

7. Alipay. Alipay: Readtime epidemic status platform online, incorporate hospital registration and other services. https://m.ithome.com/html/470168.htm, 2020 (accessed 10 February 2020).

8. Xinhuanet. Tencent releases COVID-19 service platform to enable health commissions' response services. http://www. xinhuanet.com/tech/2020-02/06/c_1125538084.htm, 2020 (accessed 6 February 2020).

9. Netease. COVID-19 Realtime Status Broadcast. https://wp.m.163.com/163/page/news/virus_report/ index.html?spssid $=61 \mathrm{db} 438402 \mathrm{ff} 850696335 \mathrm{dc}$ 6d2186b21\&amp;spsw=8\&amp;spss=other\&amp; 2020 (accessed 10 February 2020).

10. Finance Sina. Check and Be Safe. https://finance.sina.com. cn/wm/2020-02-09/doc-iimxxste9953149.shtml, 2020 (accessed 10 February 2020).

11. cn-healthcare. SuriBot, is COVID-19 a catalyst for Internet+ health care development. https://www.cn-healthcare.com/ articlewm/20200224/wap-content-1090153.html, 2020 (accessed 25 February 2020).

12. Zhihu. Manniu APP from Wonders in action to fight epidemic, use AI to build first line of defense for risk screening of COVID-19. https://zhuanlan.zhihu. com/p/106401840, 2020 (accessed 15 February 2020).

13. cn-Healthcare. Start the digital epidemic prevention and control battle! Big data, AI and cloud computing become new measures across China to fight epidemic. https://www. cn-healthcare.com/article/20200204/wap-content-529877. html, 2020 (accessed 4 February 2020).

14. Tsinghua University. Use system developed by Tsinghua for COVID-19 infection screening at home. https://news. tsinghua.edu.cn/info/1044/77400.htm, 2020 (accessed 2 February 2020).

15. Hangzhou Daily. A Zhejiang taxi driver diagnosed with COVID-19, 200 close contacts found. https://xw.q9.com/ cmsid/20200214A04PR100, 2020 (accessed 14 February 2020).

16. Xinhua News. Starting today, passengers to provide mobile phone numbers for train ticket purchase. https://mp.weixin. q9.com/s/WM7rGIDScgd3s2q_QZJXSA, 2020 (accessed 1 February 2020).

17. Huanqiu. Baidu AI temperature taking technology launched in Beijing, with an error margin only $0.05^{\circ} \mathrm{C}$. https://3w.huanqiu.com/a/15ea85/3wry6Q4IA19?agt=18,
2020 (accessed 4 February 2020).

18. people.cn. Engineers enhance drone temperature taking accuracy up to $\pm 5^{\circ} \mathrm{C}$ with a cotton swab. http://it.people. com.cn/n1/2020/0219/c1009-31594934.html, 2020 (accessed 21 February 2020).

19. Sina News. Yichun, Jiangxi: Thermal imaging drones taking body temperature. https://k.sina.cn/article_1699432410_ m654b47da03300n18e.html, 2020 (accessed 1 January 2020).

20. New healthcare. Fight COVID - 19, HIT companies in action. https://mp.weixin.q9.com/s/wSnkVgkFXHpHfVaOdoSwg, 2020 (accessed 1 January 2020).

21. cn-Healthcare. PKU-HIT develop fever clinic consultation app deployable in 6 hours, free to all medical institutions. https://www.cn-healthcare.com/articlewm/20200129/wapcontent-1084638. html, 2020 (accessed 29 January 2020).

22. CSDN. Huawei Cloud launches COVID-19 AI auxiliary diagnostic service, CT quantification results yielded in seconds. https://blog.csdn.net/devcloud/article/ details/104263141, 2020 (accessed 11 February 2020).

23. Shi Jing. AI system lends helping hand in virus diagnosis. http://www.chinadaily.com.cn/a/202002/05/ WS5e3a7b68a3101282172752d7.html, 2020 (accessed 5 February 2020).

24. Winning Healthcare. Winning Healthcare reinforces Wuhan Huoshenshan Hospital with hasty deployment of tele-medicine platform. http://www.winning.com.cn/news/ index.php?id=242, 2020 (accessed 6 February 2020).

25. Publicity Department of Huangpu District, Guangzhou Municipality. App helps people in Huangpu district report their health status and daily needs with ease. https:// baijiahao. baidu.com/s?id=1657303594618422043\&wfr=spi der\&for=pc, 2020 (accessed 1 February 2020).

26. XK Tech. Alipay to launch a national health code, happily go green. https://www.sohu.com/a/373418765_100028490, 2020 (accessed 16 February 2020).

27. Information Office of Shanghai Municipal Government. Shanghai municipal government service center launches Shanghai Health QR Code, only green codes allowed to enter. http://www.shio.gov.cn/sh/xwb/n782/n783/ u1ai23654.html, 2020 (accessed 24 February 2020).

28. Sohu. COVID-19: Time of test, Baidu Map migration big data + realtime traffic platform hands out 2.24 bil service score card. https://www.sohu.com/a/385781279_100160886, 2020 (accessed 11 April 2020).

29. Jiantao Zhang. Nankai University team predicts COVID-19 spread with big data. https://stat.nankai.edu.cn/2020/0216/ c12323a265121/page.htm, 2020 (accessed 7 February 2020).

30. Alibaba. To accelerate COVID-19 vaccine R\&D, Aliyun offers free access to all its AI computing power. https://yq.aliyun. com/articles/743585, 2020 (accessed 4 February 2020).

31. Zaker News. For chronic disease patients whose meds are disrupted by COVID-19. http://www.myzaker.com/ article/5e45e41b8e9f0944db2a3886/, 2020 (accessed 14 
February 2020).

32. Tencent News. Nearly 1,000 hospitals went online in less than a month after deploying EMR system for Huoshenshan and Leishenshan Hospitals. https://new.q9.com/rain/ a/20200212A0SJ9X, 2020 (accessed 12 February 2020).

33. Sohu. Ewell MDT \& tele-medicine platform reinforces fight against COVID-19, experts conference 'faceto-face' to discuss critical cases. https://www.sohu. com/a/371011633_756178, 2020 (accessed 21 February 2020).

34. Sina Finance. PingAn GoodDoctor releases 2019 Sustainable Development Report, platform APP received 1.11 billion visits during COVID-19. http://finance.sina.com. cn/stock/relnews/hk/2020-02-17/doc-iimxyqvz3566199. shtml, 2020 (accessed 17 February 2020).

35. China National Radio. Medical Insurance 12 Regulations back Shanghai's COVID-19 response efforts, Medical Insurance to cover Internet+ healthcare services. http:// news.cnr.cn/native/city/20200223/t20200223_524987966. shtml, 2020 (accessed 23 February 2020).

36. ifeng. Smart medicine, remote diagnosis... China Mobile's healthcare IT on frontline to fight COVID-19. http://finance. ifeng.com/c/7tncva31Cj2, 2020 (accessed 4 February 2020).

37. Chinadaily. West China Hospital of Sichuan University runs multidisciplinary $5 \mathrm{G}$ tele-conference with frontline hospitals in Wuhan. https://tech.chinadaily.com.cn/a/202002/21/ WS5e4f2fa2a3107bb6b57a132f.html, 2020 (accessed 21 February 2020).

38. China National Radio. The First Affiliated Hospital, Sun Yatsen University: Smartest minds join forces to save critical patients in Wuhan. http://www.cnr.cn/gd/gdkx/20200221/ t20200221_524986035.shtml, 2020 (accessed 21 February 2020).

39. Sina. Alibaba opens up online classroom feature for students across China and offers free telecommuting to 100 mil companies. http://k.sina.com.cn/ article_213815211_0cbe8fab02000u27g.html, 2020 (accessed 3 February 2020).

40. Sohu. Corporate WeChat releases group livestreaming feature to enable tele-education in chat groups during epidemic. https://www.sohu.com/a/369966218_100172864, 2020 (accessed 5 February 2020).

41. TroilaDJ. Trolia Cloud computing to fight epidemic with technology. https://mp.weixin.q9.com/s/acdOMKQyH7IW4QYzAXdNw, 2020 (accessed 3 February 2020).

42. Dayu Song. Tencent News. Mengcheng: Hardcore epidemic response with drones. https://view.inews.99.com/ k/20200203A046D000, 2020 (accessed 3 February 2020).

43. Tencent iNews. Meituan contact-free delivery launched in 184 cities, to cover whole country this week. https:// view.inews.99.com/w2/20200129A04E2R00?tbkt=F \&strategy $=$ \&openid $=004$ IBALMrLyGDxbWNOPoDM1I fG-s\&uid=\&refer=wx_hot, 2020 (accessed 29 January 2020).

44. Sohu. Afraid of infection through take-away delivery?Ele'me launches contact-free delivery and Safe Delivery card. https://www.sohu.com/a/369768437_120046696, 2020 (accessed 31 January 2020).

45. Sina Tech. Robot warriors in epidemic: Fight virus and protect lives. https://tech.sina.cn/2020-02-06/detailiimxyqvz0647075.d.html?ivk_sa=1023197a, 2020 (accessed 6 February 2020).

46. Sina Finance. Huoshenshan Hospital to hand off to military, watch the Huoshen speed in 140s video. http://finance.sina. com.cn/wm/2020-02-02/doc-iimxxste8242260.shtml, 2020 (accessed 2 February 2020).

47. e23. Watch together. $5 \mathrm{G}$ livestreaming takes you to construction site of Wuhan Leishenshan Hospital. https:// news.e23.cn/guonei/2020-01-30/2020013000086.html, 2020 (accessed 30 January 2020).

48. Sina Finance. $5 \mathrm{G}$ implementation completed for Wuhan Leishenshan Hospital, advancing 5G+smart medical care. http://finance.sina.com.cn/stock/relnews/us/2020-01-28/ doc-iihnzhha5151038.shtml, 2020 (accessed 2 February 2020).

49. People News. Using IoT and other advanced technologies to create a series of biosafety credibility platform products. http://www.elecfans.com/d/1168962.html, 2020 (accessed 6 February 2020).

50. Souhu News. Ewell AI+IoT plays a great role in fight against COVID-19. https://www.sohu. com/a/370200682_756178?spm=smpc.author. fd-d.6.158201310158408IPcP9, 2020 (accessed 2 February 2020).

51. Chinanews. IoT helps control COVID-19 epidemic, Tencent Cloud launches smart temperature taking solution. https:// www.chinanews.com/business/2020/02-25/9104312.shtml, 2020 (accessed 25 February 2020).

52. World Health Organization. WHO Coronavirus (COVID-19) Dashboard With Vaccination Data. https://covid19.who.int/ region/wpro/country/cn, 2021 (accessed 15 March 2021). 
TO CITE THIS ARTICLE:

Liu J, Wang Z, Huang S, Ren A. An Overview of Healthcare Information Technologies Used to Combat the COVID-19 Pandemic in China. International Journal of Digital Health. 2021; 1(1): 14, 1-9. DOI: https://doi.org/10.29337/ijdh.37

Submitted: 28 January 2021 Accepted: 23 May 2021 Published: 11 June 2021

COPYRIGHT:

(c) 2021 The Author(s). This is an open-access article distributed under the terms of the Creative Commons Attribution-NonCommercial 4.0 International License (CC-BY-NC 4.0), which permits unrestricted distribution, reproduction and adaptation in any medium, provided the original author and source are credited, and that the material is not used for commercial purposes.

See https://creativecommons.org/licenses/by-nc/4.0/.

International Journal of Digital Health is a peer-reviewed open access journal published by IJS Publishing Group. 